Keywords: TMPRSS2-ERG gene rearrangement; androgen receptor; PKA; cAMP; phosphodiesterase; androgen-independence; prostate cancer progression

\title{
Human phosphodiesterase 4D7 (PDE4D7) expression is increased in TMPRSS2-ERG- positive primary prostate cancer and independently adds to a reduced risk of post-surgical disease progression
}

\begin{abstract}
R Böttcher ${ }^{1,7}$, D J P Henderson ${ }^{2,7,8}, \mathrm{~K}$ Dulla $^{3}$, D van Strijp ${ }^{3}$, L F Waanders ${ }^{3}, \mathrm{G} \mathrm{Tevz}{ }^{3,4}, \mathrm{M} \mathrm{L} \mathrm{Lehman}^{4}$, D Merkle ${ }^{3}$, G J L H van Leenders ${ }^{5}$, G S Baillie ${ }^{2}$, G Jenster ${ }^{1}$, M D Houslay ${ }^{6}$ and R Hoffmann ${ }^{\star, 2,3}$

${ }^{1}$ Department of Urology, Erasmus Medical Center, Rotterdam 3000 CA, The Netherlands; ${ }^{2}$ Institute of Cardiovascular and Medical Science, University of Glasgow, Glasgow G12 8TA, Scotland; ${ }^{3}$ Departments of Oncology Solutions and Precision Diagnostics, Philips Research Europe, Eindhoven 5656 AE, The Netherlands; ${ }^{4}$ Australian Prostate Cancer Research Centre-Institute of Health and Biomedical Innovation, University of Technology, and Translational Research Institute, Brisbane, Queensland 4102, Australia; ${ }^{5}$ Department of Pathology, Erasmus Medical Center, Rotterdam 3000 CA, The Netherlands and ${ }^{6}$ Institute of Pharmaceutical Science, King's College London, London WC2R 2LS, UK
\end{abstract}

Background: There is an acute need to uncover biomarkers that reflect the molecular pathologies, underpinning prostate cancer progression and poor patient outcome. We have previously demonstrated that in prostate cancer cell lines PDE4D7 is downregulated in advanced cases of the disease. To investigate further the prognostic power of PDE4D7 expression during prostate cancer progression and assess how downregulation of this PDE isoform may affect disease outcome, we have examined PDE4D7 expression in physiologically relevant primary human samples.

Methods: About 1405 patient samples across 8 publically available qPCR, Affymetrix Exon 1.0 ST arrays and RNA sequencing data sets were screened for PDE4D7 expression. The TMPRSS2-ERG gene rearrangement status of patient samples was determined by transformation of the exon array and RNA seq expression data to robust z-scores followed by the application of a threshold $>3$ to define a positive TMPRSS2-ERG gene fusion event in a tumour sample.

Results: We demonstrate that PDE4D7 expression positively correlates with primary tumour development. We also show a positive association with the highly prostate cancer-specific gene rearrangement between TMPRSS2 and the ETS transcription factor family member ERG. In addition, we find that in primary TMPRSS2-ERG-positive tumours PDE4D7 expression is significantly positively correlated with low-grade disease and a reduced likelihood of progression after primary treatment. Conversely, PDE4D7 transcript levels become significantly decreased in castration resistant prostate cancer (CRPC).

Conclusions: We further characterise and add physiological relevance to PDE4D7 as a novel marker that is associated with the development and progression of prostate tumours. We propose that the assessment of PDE4D7 levels may provide a novel, independent predictor of post-surgical disease progression.

${ }^{*}$ Correspondence: Dr R Hoffmann; E-mail: ralf.hoffmann@philips.com

${ }^{7}$ These authors contributed equally to this work.

${ }^{8}$ Current address: The Salk Institute, Regulatory Biology Lab, La Jolla, CA 92307, USA.

Received 29 May 2015; revised 14 August 2015; accepted 20 August 2015

(c) 2015 Cancer Research UK. All rights reserved 0007-0920/15

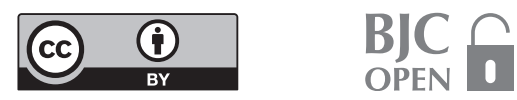


Prostate cancer is the most commonly occurring non-skin malignancy in men, with an estimated 900000 new cases diagnosed world-wide in 2013 (Ferlay et al, 2015). However, reactive clinical intervention after routine diagnosis often leads to significant overtreatment of non-aggressive tumours. This has severe negative impacts on both patient quality of life and the medical resources of healthcare institutions (Andriole et al, 2009; Schröder et al, 2009). Therefore, the characterisation of new biomarkers and methods of clinical assessment is of significant importance when assessing the need for different forms of clinical intervention.

Previous studies have shown that signalling pathways mediated by the second messenger cAMP have various roles in the development and progression of prostate cancer (Merkle and Hoffmann, 2011). Cyclic nucleotide phosphodiesterases (PDEs) (Conti and Beavo, 2007; Maurice et al, 2014) provide the sole means of degrading cAMP and cGMP in cells, and are pivotally placed to regulate cAMP signalling by virtue of their intracellular location and post-translational modification (Lugnier, 2006; Houslay, 2010). Each of the 11 PDE genes encode for a series of isoform variants, thereby greatly increasing the diversity of unique regulatory mechanisms, intracellular targeting and kinetic properties, which define functionally independent and unique signalling roles within the cell (Houslay et al, 2007; Houslay, 2010; Francis et al, 2011). This diversity underpins a paradigm of compartmentalised, temporally gated cyclic nucleotide signalling. Due to the complexity of these orchestrated signalling events, any change in $\mathrm{PDE}$ isoform expression or regulation can functionally contribute to disease onset (Lee et al, 2012; Michot et al, 2012; Apuhan et al, 2013; Kaname et al, 2014; Yoon et al, 2014). The molecular characterisation of these changes can be expected to provide means for the development of novel therapeutics and diagnostics (Houslay et al, 2005; Houslay, 2010).

Members of the PDE4D subfamily have been implicated as underpinning the molecular pathology of various diseases including prostate cancer (Rahrmann et al, 2009; Henderson et al, 2014), stroke (Gretarsdottir et al, 2003), acrodysostosis (Kaname et al, 2014) and COPD (Yoon et al, 2014). The PDE4D gene encodes a cohort of isoforms that are classified as long, short and super-short. Long isoforms possess two conserved regulatory domains, called UCR1 and UCR2, which allow long isoforms to be phosphorylated and activated by PKA $\left(3^{\prime}, 5^{\prime}\right.$ cAMP-dependent protein kinase) after cAMP elevation in cells (Hoffmann et al, 1998), as well as being functionally regulated through phosphorylation by activated forms of ERK, MK2 and AMPK (MacKenzie et al, 2011; Sheppard et al, 2014).

PDE4D7 is a long isoform member of this subfamily (Wang et al, 2003). We have demonstrated that PDE4D7 exhibits a specific pattern of intracellular localisation in prostate cancer cells, where it is functionally targeted to the sub-plasma membrane compartment (Henderson et al, 2014). Spatially constrained PDE4D7 appears to perform a pivotal role in these cells by desensitising sub-plasma membrane-localised cAMP signalling (Henderson et al, 2014), as well as providing a node for crosstalk with signalling pathways that elicit the activation of Erk, MK2 and AMPK (Hoffmann et al, 1999; Baillie et al, 2000; MacKenzie et al, 2011; Sheppard et al, 2014). PDE4D7 activity is also regulated by PKA phosphorylation within its unique $\mathrm{N}$-terminal region (Byrne et al, 2015). Interestingly, susceptibility markers for ischaemic stroke also map to the region of Chr5q12, where PDE4D7 and the androgen-regulated PART1 exons are located (Gretarsdottir et al, 2003).

We have previously demonstrated that PDE4D7 is highly expressed in androgen-responsive prostate cancer cell lines and xenografts, while being downregulated in castration resistant samples (Henderson et al, 2014). Indeed, the ectopic overexpression of PDE4D7 in castration resistant prostate cancer (CRPC) cell lines reduced cellular proliferation, while specific knockdown of the PDE isoform in androgen-sensitive cells lead to an increase in cellular proliferation, indicating a functional role of PDE4D7 downregulation during the progression to CRPC growth. Here, we set out to assess whether the changes in PDE4D7 expression we observed in model systems have clinical relevance. To do this, we analysed 1405 tumour samples sourced from 8 independent patient cohorts that were enroled at different clinical centres (Supplementary Table 1). Our analyses of clinical samples highlight an increase in PDE4D7 expression during initial tumorigenesis and further support our contention that PDE4D7 levels then fall profoundly in CRPC, suggesting that PDE4D7 transcripts may provide a potentially useful biomarker and therapeutic target.

\section{MATERIALS AND METHODS}

Human tissue samples. Human tissues samples were obtained under local laws and regulation to obtain and handle patient material for research purposes. Sample descriptions are depicted in Figure 1A.

Molecular biology (RNA extraction, cDNA synthesis and primer design). If not otherwise indicated RNA isolation, cDNA conversion and Real-Time PCR were performed using RNeasy Kit (QIAGEN GmbH, Hilden, Germany, 74004), iScript cDNA synthesis kit (Bio-Rad Inc, Hercules, CA, USA, 170-8890), GeneAmp Fast PCR Master Mix (Applied Biosystems Inc, Foster City, CA, USA, 4362070) respectively, according to the manufacturer's instruction. Real-Time PCR probe and primer sets were developed by targeting isoform-specific intron-spanning regions of genetic code (Supplementary Table 3).

Quantitative RT-PCR (qRT-PCR). To enable the comparison of qPCR data across different experiments, we normalised the $\mathrm{Ct}$ value for PDE4D7 against the mean of the $\mathrm{Ct}$ values for the reference genes (Supplementary Table 3 ) to generate a normalised PDE4D7 expression value.

We use the following formula to normalise the raw $\mathrm{Ct}$ values:

$$
N\left(\mathrm{Ct}_{\text {gene of interest }}\right)=\operatorname{Mean}\left(\mathrm{Ct}_{\text {ref gene }}\right)\left(\mathrm{Ct}_{\text {gene of interest }}\right)
$$

Where $\mathrm{N}\left(\mathrm{Ct}_{\text {gene of interest }}\right)=$ is normalised gene expression value for a gene of interest; where Mean $\left(\mathrm{Ct}_{\text {ref gene }}\right)$ is the arithmetic mean of the PCR Cq values of the selected combination of reference genes; where $\left(\mathrm{Ct}_{\text {gene of interest }}\right)$ is the PCR Cq value of the gene of interest.

Note: in case DNA microarray or RNA seq technologies was used to measure PDE4D7 expression, the qPCR Ct value was replaced by a normalised measurement of the respective technology, for example, an robust multi-array average (RMA) normalised gene expression value for DNA microarrays, or a TPM (transcript per million) normalised gene expression value for RNA sequencing.

Analysis of affymetrix human exon arrays. Raw CEL files were downloaded from Gene Expression Omnibus for the publically available data sets (Supplementary Table 1). Data processing and RMA normalisation were performed using the aroma.affymetrix R-package (Affymetrix Inc, Santa Clara, CA, USA; Purdom et al, 2008) and transcript isoform expression was measured by averaging $\log 2$-transformed intensity values of the following isoform-specific probe sets: PDE4D7 (2858406, 2858407 and 2858408); Note: for data set Erho et al (2013) (Supplementary Table 1) only probe set 2858408 was used in the analysis as probe sets 2858406 and 2858407 showed relatively limited signal intensities compared with probe set 2858408 .

RNA seq data analysis. RNA seq data of 193 prostate cancer clinical samples (36 normal, 157 tumour) was downloaded from The Cancer Genome Atlas (TCGA) Data Portal (4 September 2013) 
and the expression value of genes and isoforms (TPM-transcript per million) was estimated as previously described (Li et al, 2010).

Positive TMPRSS2-ERG fusion status was estimated in general by transformation to robust $z$-scores. Positive TMPRSS2-ERG fusion status was estimated by transformation to robust $z$-scores, utilising robust statistical measures, namely median and median absolute deviation, to replace mean and s.d., which are sensitive to outliers. Thus, $\log 2$-transformed expression values were converted by $z$-score $=($ expression - median $($ expression $)) /(\operatorname{MAD}($ expression $))$, and a threshold of $>3$ was applied to define samples with positive fusion events. Subsequently, a threshold of $>3$ was applied to define samples with positive fusion events. For the Erho et al (2013) data set, we applied a supervised clustering algorithm (Partitioning Around Medoids) to assign prostate cancer samples in one of the two clusters (high ERG or low ERG) based on the $\log 2$-transformed expression values of ERG. High ERG expression was subsequently assumed as representative for the presence of a positive TMPRSS2-ERG fusion event.

To assess whether any evidence of ERG binding in the genomic region of PDE4D could be observed, we utilised public ChIP-seq data (GSE14092) from the VCaP prostate cancer cell line after liftOver (https://genome.ucsc.edu/cgi-bin/hgLiftOver) to hg19 and found 43 peaks overlapping PDE4D when including 50-kb flanking regions. One of these peaks overlapped the PDE4D7 promoter region, while another was located in close proximity $(<200$ bases distance), which may hint towards an involvement of ERG binding in regulation of PDE4D7 expression.

Statistical data analysis. For ROC analysis, calculation of AUC under the ROC, ROC $P$-values and Box-and-Whisker plots the statistical software package MedCalc (MedCalc Software BVBA, Ostend, Belgium) was used. $P$-values for differences of mean expression were calculated by using Wilcoxon-Mann-Whitney testing unless mentioned otherwise.

Kaplan-Meier Survival curves have been generated by the medical statistical software package MedCalc based on the time to event for those patients who experienced the respective event (e.g., biochemical recurrence (BCR) or clinical recurrence (CR) of disease after surgery) and for those patients who did not suffer from the event at the time of follow-up (censored data). Further, to segregate the analysed patient cohort into two survival groups we determined a cut-off of PDE4D7 expression from a ROC curve analysis. The respective cut-off was objectively determined from the ROC curve at the unique point in the curve, where the sum of sensitivity and specificity reached a maximum.

\section{RESULTS}

We have recently provided evidence, suggesting that PDE4D7 may play an important role in regulating cAMP signalling during prostate cancer progression (Henderson et al, 2014). To further explore this finding, we have evaluated the expression of PDE4D7 in a total of eight clinically relevant patient data sets. These data sets comprised a total of 1405 patient samples stratified into 8 sample categories listed in Figure 1A. Three different technology platforms were also leveraged to ensure reproducibility and significance of the gene expression data for PDE4D7, namely: (1) qPCR; (2) Affymetrix Human Exon Array 1.0 ST; (3) RNA seq (see Supplementary Table 1). More details of the data sets used within this study can be found in Supplementary Tables 1 and 2 .

PDE4D7 expression correlates with primary localised prostate tumours and is significantly downregulated in CRPC. Our previous investigation in cell lines and xenograft material found that PDE4D7 was differentially expressed between androgen sensitive/responsive and CRPC cells (Henderson et al, 2014). To assess if this finding is physiologically relevant, we thought it prudent to examine PDE4D7 transcript expression in primary patient samples. We selected three prostate cancer exon array data sets (Taylor et al, 2010; Boormans et al, 2013; Böttcher et al, 2015; J Schalken, Radboud Uinversity Nijmegen Medical Center, Nijmegen, The Netherlands, Personal Communication; Supplementary Table 1) and analysed a range of primary prostate cancer samples including tissues collected from patients who developed biochemical or clinical tumour progression after primary treatment, as well as metastases and CRPC (Figure 1B-D; Supplementary Table 5). We observed a striking downregulation in PDE4D7 expression between primary prostate cancer without tumour progression (Primary PCa, NP) and primary prostate cancer tissue with either progression to $\mathrm{BCR}$ (Primary PCa, BCR) or CR (Primary PCa, CR). The ROC analysis for the group-wise comparisons revealed AUCs are between 0.61 and 0.82 (Supplementary Table 5). In line with our previous findings, the most significant downregulation was observed between tissues representing primary prostate cancer vs CRPC (data sets Taylor et al, 2010 and J Schalken, Personal Communication; $P$-values for differential PDE4D7 expression 5.80E - 04, and $1.90 \mathrm{E}-05$, respectively; AUCs for PDE4D7 ROC analysis $0.82,95 \%$ CI $0.73-0.88$ and $0.81,95 \%$ CI $0.71-0.90$, respectively; Supplementary Table 5). In contrast to the comparison between primary tumours and CRPC, a differential expression of PDE4D7 between primary prostate cancer and metastatic tissue could not be confirmed in the data set from Taylor et al (2010) $(P=1.60 \mathrm{E}-01 ; \mathrm{AUC}=0.65 ; 95 \%$ CI $0.55-0.74)$ nor in Boormans et al (2013); Böttcher et al (2015) $(P=1.10 \mathrm{E}-01$; AUC $=0.67$; 0.49-0.82); however, in the data set produced by J Schalken, Personal Communication the expression difference was significant $(P=4.6 \mathrm{E}-04)$ with a very large AUC $(0.91 ; 95 \%$ CI $0.81-0.97)$. Overall this data confirms our original observation made in in vitro models of prostate cancer; PDE4D7 is significantly downregulated in aggressive and advanced forms of prostate cancer.

PDE4D7 expression is upregulated in localised primary prostate tumours and correlates with TMPRSS2-ERG gene fusion. To assess the significance of PDE4D7 expression within the context of the normal prostate epithelia, we extended the exon array analysis to include patient tissue taken from areas adjacent to prostate tumours (NAT). We examined 850 patient samples across seven independent data sets (Supplementary Tables 1 and 2). Interestingly, we observed a significant upregulation of PDE4D7 in primary prostate cancer vs NAT (Figure 2A-D; Supplementary Table 6). This suggests that PDE4D7 upregulation in prostate tissue may be involved with initial tumorigenesis.

To investigate this further, we set out to establish if there was any correlation between PDE4D7 expression and factors known to regulate initial tumorigenesis in the prostate. The TMPRSS2-ERG gene fusion has previously been reported as a clinical indicator for prostate cancer formation. Since its discovery, this prostate cancerspecific fusion event has been described in $\sim 50 \%$ of prostate cancer patients and has become a molecular hallmark of prostatic tumours (Tomlins et al, 2005). Given the status of TMRSS2-ERG as the most relevant genomic fusion event so far identified in prostate cancer, we tested the expression of PDE4D7 in 1106 patients with (Primary PCa, TMPRSS2-ERG positive; Figure 3) and without (Primary PCa, TMPRSS2-ERG negative; Figure 3) this gene fusion. Figure $3 \mathrm{~A}-\mathrm{C}$ shows the PDE4D7 expression across three exon array data sets (data sets Taylor et al, 2010; Brase et al, 2011; Boormans et al, 2013; Böttcher et al, 2015; Supplementary Table 1), which we picked for graphical illustration (all data sets where we had information on TMRPSS2-ERG rearrangement information available can be found in Supplementary Table 7). Intriguingly, we observed a significantly higher PDE4D7 expression in tumour samples that harboured the TMPRSS2-ERG gene fusion when compared with TMPRSS2-ERG negative samples or when compared with NAT (2-fold median increase, with some samples in 
A

\begin{tabular}{|l|l|}
\hline Category & Description \\
\hline NAT & Normal adjacent tissue \\
\hline BL & Benign lesions due to infection or inflammation \\
\hline BPH & Benign prostate hyperplasia \\
\hline Primary PCa, NP & Primary tumour, no progression \\
\hline Primary PCa, BCR & Primary tumour, biochemical recurrence \\
\hline Primary PCa, CR & Primary tumour, clinical recurrence \\
\hline Metastases & Metastatic tissue (e.g. lymph node) \\
\hline CRPC & Castration resistant prostate cancer \\
\hline
\end{tabular}

C

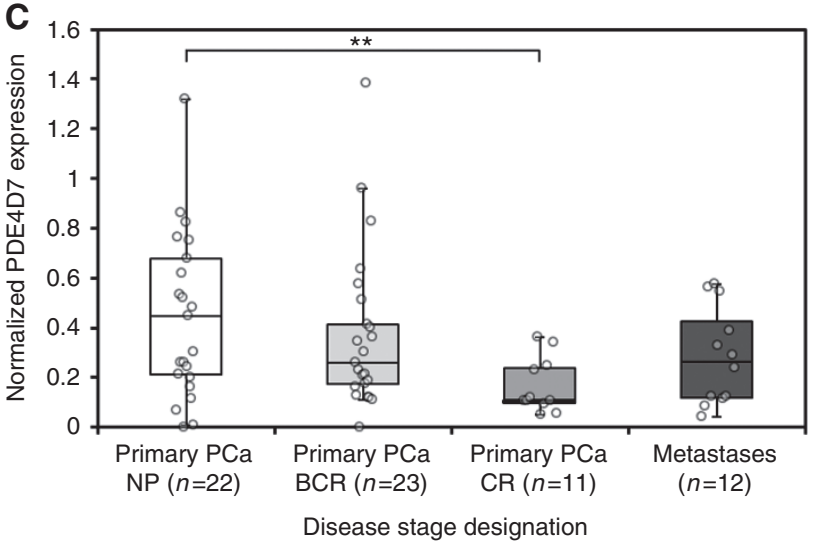

B

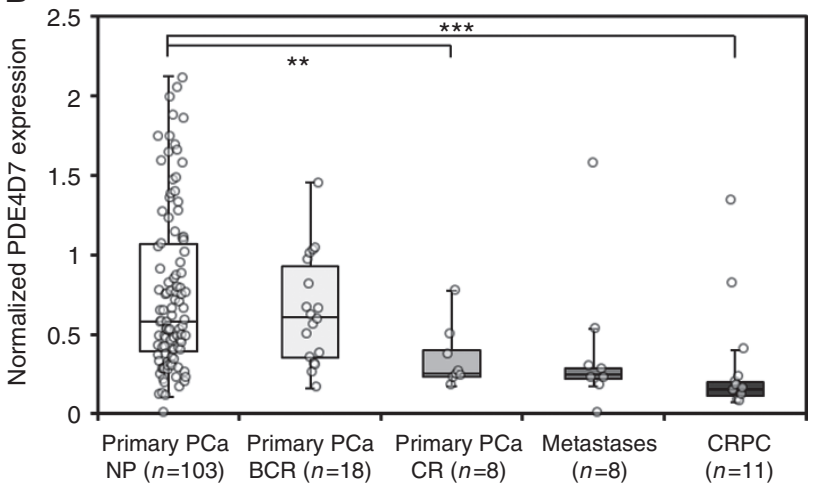

Disease stage designation

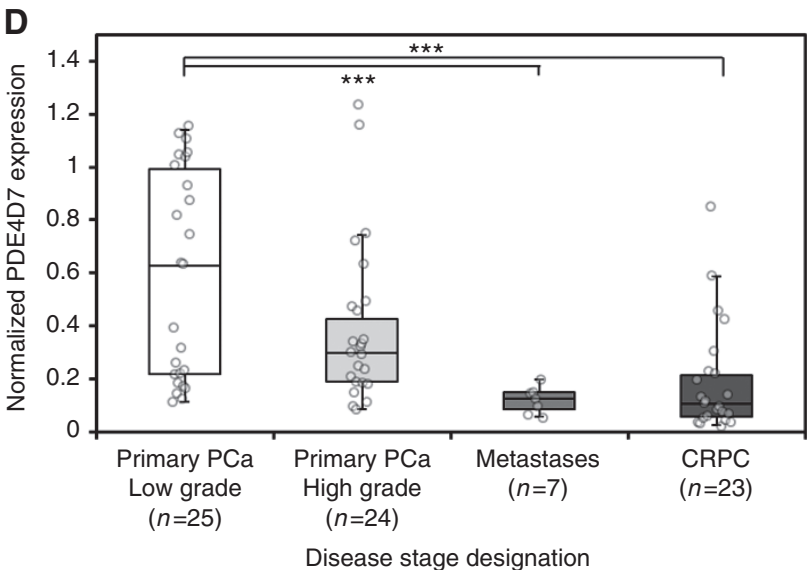

Figure 1. Expression of PDE4D7 splice variant in primary, metastatic and castration resistant cancerous prostate tissues. Box-and-Whisker plots of the normalised PDE4D7 transcript expression in various prostate cancer tissues. For all data sets, and all $P$-values and AUCs see Supplementary Table 5. (A) Disease stage annotation for this study. (B) Data: Taylor et al, 2010; P-values of group comparison for difference of mean PDE4D7 expression: (Primary PCa, NP) vs (Primary PCa, BCRandCR), $P=7.2 \mathrm{E}-02$; (Primary PCa, NP\&BCR) vs (Primary PCa, CR), $P=5.90 E-03 ;(P r i m a r y$ $P C a$, all) vs (Metastases), $P=1.60 E-01$; (Primary PCa, all) vs (CRPC), $P=5.8 E-04 ;(C)$ Data: Boormans et al, 2013; Böttcher et al, 2015; $P$-values of group comparison for difference of mean PDE4D7 expression: (Primary PCa, NP) vs (Primary PCa, BCR\&CR), P=6.50E - 02; (Primary PCa, NP) vs (Primary PCa, CR), $P=1.30 E$ - 03; (Primary PCa, all) vs (Metastases), $P=1.1 \mathrm{E}-01$; (D) Data: J Schalken, Personal Communication; $P$-values of group comparison for difference of mean PDE4D7 expression: (Primary PCa, low grade) vs (Primary PCa, high grade), $P=2.0 E-01$; (Primary PCa, all grades) vs (Metastases), $P=4.60 \mathrm{E}-04$; (Primary $\mathrm{PCa}$, all grades) vs (CRPC), $P=1.90 \mathrm{E}-05$. ${ }^{\star \star} P<0.01$ and ${ }^{\star \star \star} P<0.001$.

excess of 30-fold upregulation; $P$-values of group-wise comparisons between TMPRSS2-ERG negative $v s$ positive tumours: $3.33 \mathrm{E}-08$; $8.60 \mathrm{E}-03 ; 3.80 \mathrm{E}-06$, respectively). At the same time there was no significant expression difference observed between TMPRSS2$E R G$ gene fusion negative cancer samples and NAT (Figure 3A-C; Supplementary Table 7).

PDE4D7 expression is positively correlated with low-grade TMPRSS2-ERG-positive prostate tumours. Having discovered a strong correlation between TMPRSS2-ERG fusion and PDE4D7 expression, we then set out to ascertain if cancer aggressiveness is correlated with PDE4D7 expression. We compared the transcript levels of PDE4D7 against pathology-graded cancer samples utilising three exon array data sets (Taylor et al, 2010; Brase et al, 2011; J Schalken, Personal Communication; Supplementary Table 1), as well as the TCGA prostate adenocarcinoma RNA seq Data Set Prostate Cancer (Release September 2013). We categorised Gleason score (pGleason) into the following four groups of increasing grade: (1) pGleason $3+3$, (2) pGleason $3+4$, (3) pGleason $4+3$, (4) pGleason $\geqslant 4+4$. A total of 264 patients were included in this stratification, and Supplementary Table 8 provides an overview of various group-wise comparisons of these different pGleason groups. Amazingly, a significant downregulation of PDE4D7 between low grade (pGleason $\leqslant 3+4) v s$ high grade (pGleason $\geqslant 4+3$ ) tumours was only observed in patients possessing the TMPRSS2-ERG gene fusion (Figure 4A and B; Supplementary Table 8). The initial increase in PDE4D7 expression in low-grade prostate cancer is in keeping with our observations from Figure 3. It is significant that in TMPRSS2ERG-positive tumour samples the expression of PDE4D7 is negatively correlated with increasing pGleason, highlighting the transient nature of PDE4D7 upregulation. This finding bears a striking resemblance to our previous observations in cell lines and xenografts (Henderson et al, 2014).

PDE4D7 expression is correlated with clinical outcome in patients expressing the TMPRSS2-ERG gene fusion. To test our hypothesis that PDE4D7 expression can predict clinical outcome in patients with positive TMPRSS2-ERG gene rearrangement, we used an exon array data sets covering 527 eligible patient samples where longitudinal outcome data was available (Taylor et al, 2010; Boormans et al, 2013; Erho et al, 2013). The data allowed for prediction of BCR after primary treatment. The patients were grouped according to their TMPRSS2-ERG gene fusion status, as well as according to pGleason (5 and 6, 7, 8, and 9 and 10). We then compared the PDE4D7 expression in patient groups with $v s$ without BCR during 5-years follow-up after primary treatment (Figure 4C and Supplementary Table 9). We could not detect a significant change in the expression of PDE4D7 in any of the TMPRSS2-ERG-negative pGleason groupings. However, for patient 
groups with positive TMPRSS2-ERG gene fusion status we found significant differential expression in the pGleason 7 group between no progression and BCR during follow-up, while this was not the case for the pGleason scores $>7$. Unfortunately, there is only a single patient sample in the pGleason 5 and 6 group with positive TMPRSS2-ERG status and progression to BCR so we could not calculate a $P$-value.
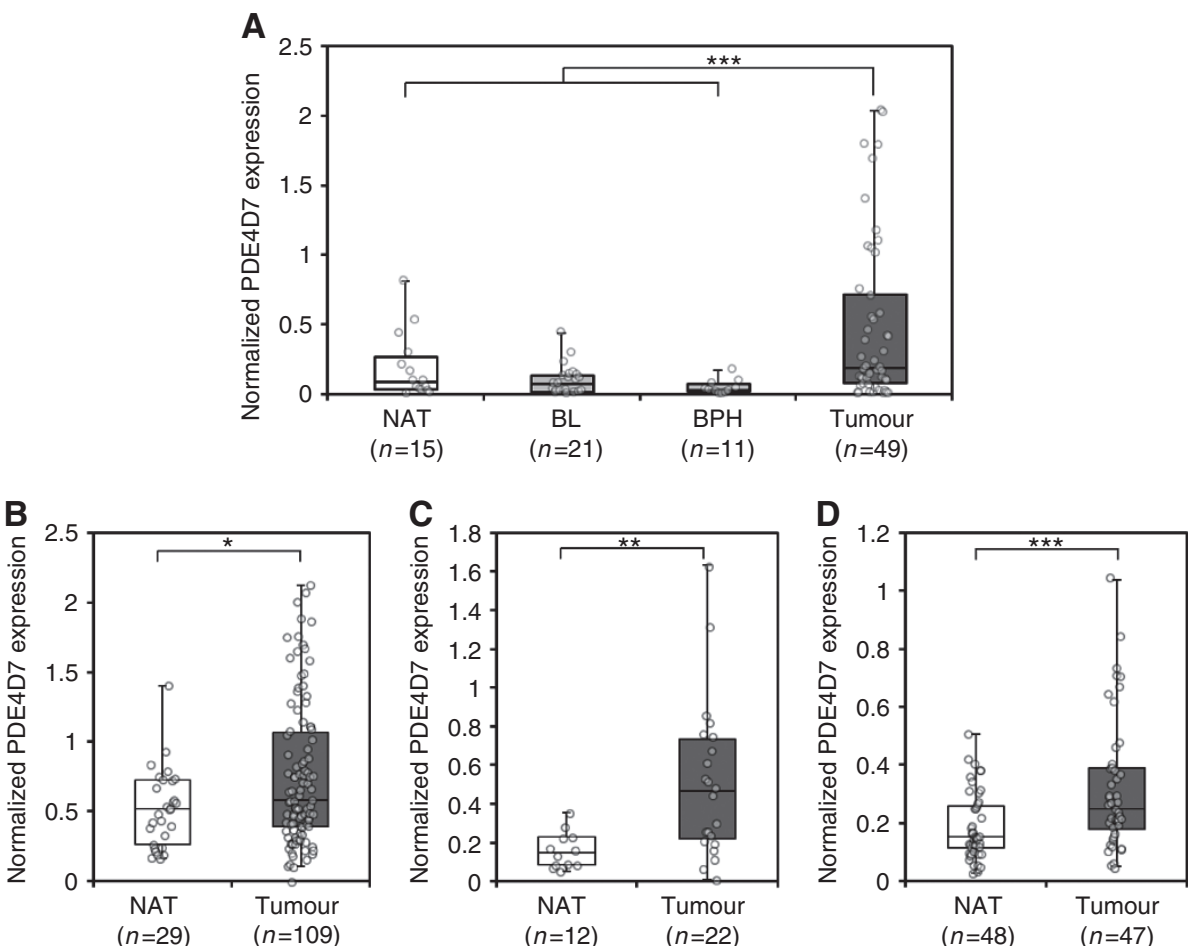

Figure 2. Expression of PDE4D7 splice variant in normal, benign vs cancerous prostate tissues. Box-and-Whisker plots of the normalised PDE4D7 transcript expression in various prostate cancer tissues. For all data sets and all P-values see Supplementary Table 6. (A) Data: Origene; $P$-values of group comparison for difference of mean PDE4D7 expression: (NAT) vs (Primary PCa), $P=6.86 E-02$; (NAT\&BL\&BPH) vs (Primary PCa), $P=1.31 \mathrm{E}-04 ;(\mathrm{BL \& BPH})$ vs (Primary PCa), $P=4.0 \mathrm{E}-04$; (BPH) vs (Primary PCa), $P=3.2 \mathrm{E}-02$; (B) Data: Taylor et al, 2010; $P$-values of group comparison for difference of mean PDE4D7 expression: (NAT) vs (Primary PCa), P=3.30E - 02; (C) Data: Boormans et al, 2013; Böttcher et al, 2015; $P$-values of group comparison for difference of mean PDE4D7 expression: (NAT) vs (Primary PCa), $P=3.50 E-03$; (D) Data: Brase et al, 2011; $P$-values of group comparison for difference of mean PDE4D7 expression: (NAT) vs (Primary PCa), $P=1.00 E-03 .{ }^{\star} P<0.05,{ }^{\star \star} P<0.01$ and ${ }^{\star \star \star} P<0.001$.

\section{A}

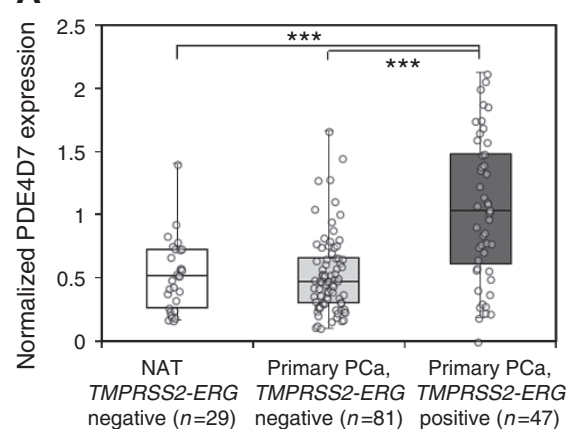

B

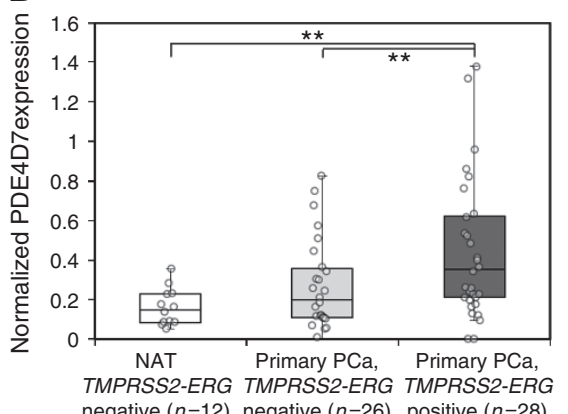

C

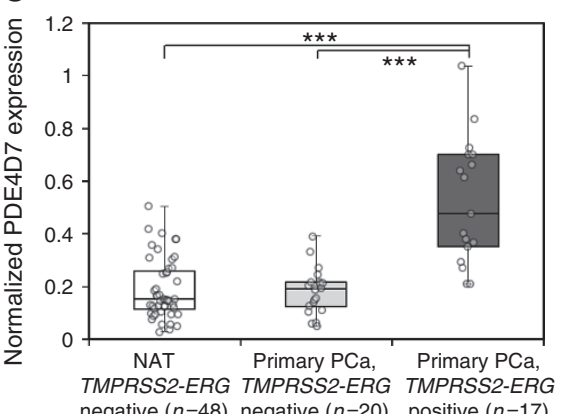

Figure 3. Correlation of PDE4D7 expression in normal and cancerous human prostate tissues to TMPRSS2-ERG gene fusion status. (A) Box-andWhisker plots of the normalised PDE4D7 transcript expression in various prostate cancer tissues. For all data sets, and all P-values see Supplementary Table 7. Positive TMPRSS2-ERG fusion status was estimated by transformation to robust z-scores (Materials and Methods). Subsequently, a threshold of $>3$ was applied to define samples with positive fusion events. Samples were divided into three different groups: (1) normal adjacent tissue without TMPRSS2-ERG fusion events (NAT TMPRSS2-ERG negative); (2) prostate tumour tissue without TMPRSS2-ERG fusion events (Primary PCa, TMPRSS2ERG negative), and (3) prostate tumour tissue with TMPRSS2-ERG fusion events (Primary PCa, TMPRSS2-ERG positive). (A) Data: Taylor et al, 2010; $P$-values of group comparison for difference of mean PDE4D7 expression: (NAT TMPRSS2-ERG negative) vs (Primary PCa, TMPRSS2-ERG negative), $P=9.00 E-01$; (NAT TMPRSS2-ERG negative) vs (Primary PCa, TMPRSS2-ERG positive), $P=1.10 \mathrm{E}-05$; (Primary PCa, TMPRSS2-ERG negative) vs (Primary PCa, TMPRSS2-ERG positive), $P=3.33 E-08$. (B) Data: Boormans et al, 2013; Böttcher et al, 2015; $P$-values of group comparison for difference of mean PDE4D7 expression: (NAT TMPRSS2-ERG negative) vs (Primary PCa, TMPRSS2-ERG negative), P=5.90E - 01; (NAT TMPRSS2ERG negative) vs (Primary PCa, TMPRSS2-ERG positive), $P=5.60 E-03$; (Primary PCa, TMPRSS2-ERG negative) vs (Primary PCa, TMPRSS2-ERG positive), $P=8.60 \mathrm{E}-03$. (C) Data: Brase et al, 2011; P-values of group comparison for difference of mean PDE4D7 expression: (NAT TMPRSS2-ERG negative) vs (Primary PCa, TMPRSS2-ERG negative), $P=7.80 E$ - 01; (NAT TMPRSS2-ERG negative) vs (Primary PCa, TMPRSS2-ERG positive), $P=5.10 E-07$; (Primary PCa, TMPRSS2-ERG negative) vs (Primary PCa, TMPRSS2-ERG positive), $P=3.80 E-06 .{ }^{* \star} P<0.01$ and ${ }^{\star \star \star} P<0.001$. 

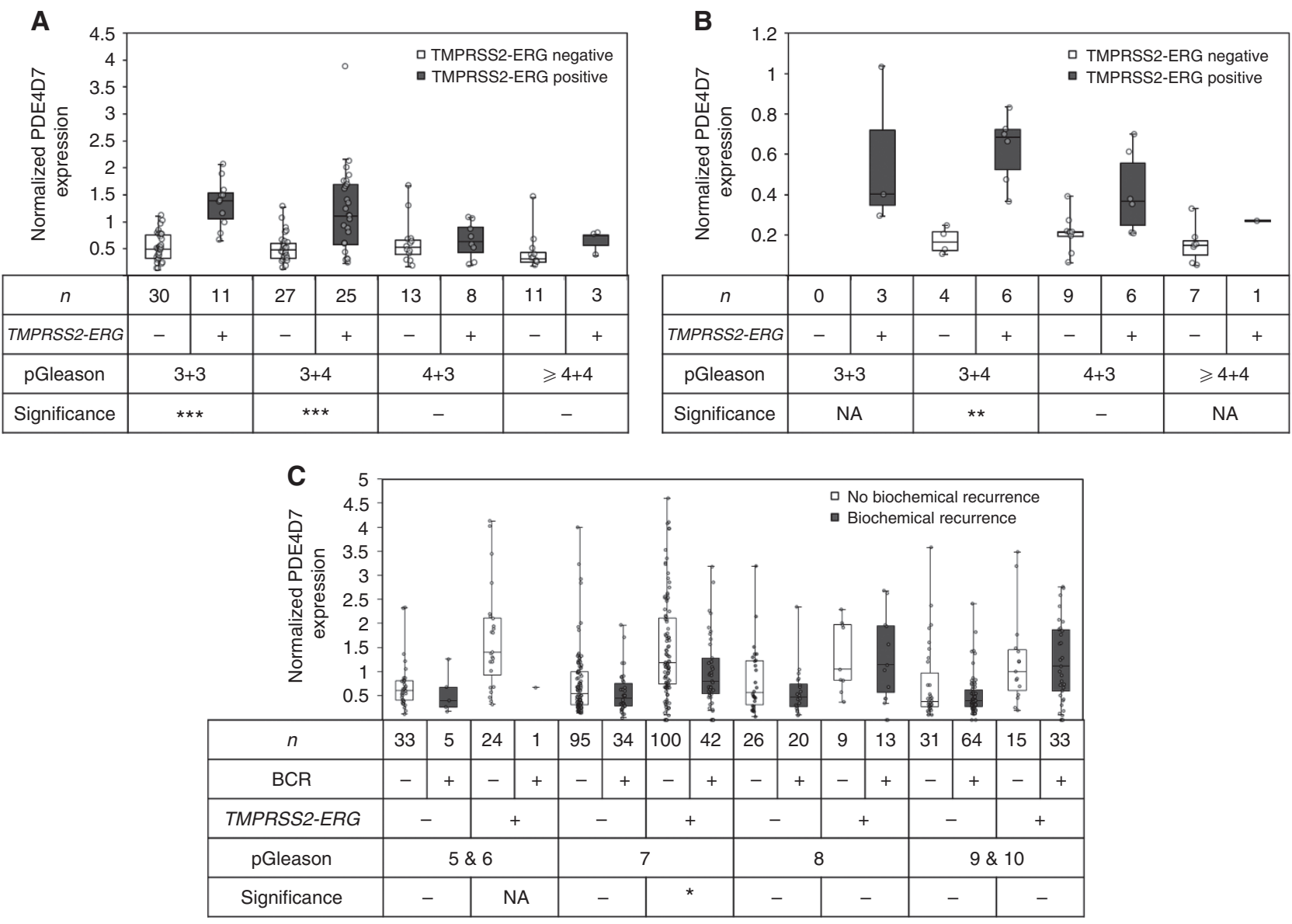

Figure 4. Correlation of PDE4D7 expression to pathology gleason score. (A and B) Box-and-Whisker plots of the normalised PDE4D7 transcript expression in various prostate cancer tissues. For all data sets, and all P-values see Supplementary Table 8 (data sets Taylor et al, 2010; Brase et al, 2011) and Supplementary Table 9 (data set Erho et al, 2013). For estimation of positive TMPRSS2-ERG fusion status see Materials and Methods. Patient cohorts were categorised according to their pGleason on histology as indicated. (A) Data Taylor et al, 2010; P-values of group comparison for difference of mean PDE4D7 expression: (pGleason $3+3 \& 3+4$ (TMPRSS2-ERG negative)) vs (pGleason $4+3 \& \geqslant 4+4$ (TMPRSS2-ERG negative)), $P=4.80 E-01$; (pGleason $3+4 \& 3+4$ (TMPRSS2-ERG positive)) vs (pGleason $4+3 \& \geqslant 4+4$ (TMPRSS2-ERG positive)), $P=2.40 E-03 ;(B)$ Data Brase et al, 2011; P-values of group comparison for difference of mean PDE4D7 expression: (pGleason $3+3 \& 3+4$ (TMPRSS2-ERG negative)) vs (pGleason $4+3 \& \geqslant 4+4$ (TMPRSS2-ERG negative)), $P=8.20 \mathrm{E}-01$; (pGleason $3+4 \& 3+4$ (TMPRSS2-ERG positive)) vs (pGleason $4+3 \& \geqslant 4+4$ (TMPRSS2-ERG positive)), $P=4.20 \mathrm{E}-02$; (C) Data Erho et al, 2013; progression after primary treatment (i.e., surgery) is indicated as $B C R(+)$ or absence $(-)$ of BCR. P-values of group comparison for difference of mean PDE4D7 expression: (pGleason 7 (TMPRSS2-ERG negative), NP) vs (pGleason 7 (TMPRSS2-ERG negative, BCR)), $P=1.10 \mathrm{E}-01$; (pGleason 7 (TMPRSS2-ERG positive), NP) vs (pGleason 7 BCR (TMPRSS2-ERG positive), $\mathrm{BCR}), P=4.60 \mathrm{E}-02$.

However, this particular sample shows a very low PDE4D7 expression value compared with the samples in this pGleason group but without post-treatment progression (Figure 4C). We concluded from this that low PDE4D7 expression values in patient samples with low pGleason scores (6 and 7) are associated with an increased likelihood of biochemical failure after primary intervention.

A graphical representation of PDE4D7 expression in various cell and tissue types including AR negative/AR positive cell lines and xenografts, primary prostate cancer with and without progression to biochemical or CR, metastases and CRPC is shown in Figure 5A (cell lines and xenograft samples) and Figure 5B (patient samples; Supplementary Table 4). The samples are ordered based on their normalised PDE4D7 expression. For the cell lines, xenografts, primary tumours without progression and primary tumours with progression to BCR or CR, as well as CRPC tumours, the status of the TMPRSS2-ERG rearrangement is indicated. In general, the more aggressive type of samples are represented by low expression levels of PDE4D7, while less aggressive samples show elevated PDE4D7 expression. It is evident from the depicted cell lines and xenografts that the expression level of PDE4D7 is largely influenced by its TMPRSS2-ERG rearrangement status rather than its AR expression status, where AR positive cell lines without gene fusion show low PDE4D7 expression, while cell lines of the same category but positive gene translocation demonstrate high PDE4D7 expression levels (Figure 5A). It is also of importance to note that this effect seems to be very specific to the ERG translocation as cell lines or xenograft samples with ETV1 or ETV4 translocations do not show elevated PDE4D7 transcription (Figure 5A). Also, looking at the samples collected from patients without disease progression during follow-up reveals that those samples that were positively tested for TMPRSS2-ERG in general show increased expression of PDE4D7 (Figure 5B). This was also the case for primary tumour samples where patients progressed to either biochemical or CR as well as for CRPC. We further annotated for patients who experienced a biochemical relapse the time to PSA recurrence as two categories-relapse $<24$ months $v s$ relapse $>24$ months after primary treatment. We observed a clear association between an increased PDE4D7 expression level and an elevated time to recurrence $(P=1.72 \mathrm{E}-02$; eight out of nine patients with normalised PDE4D7 expression $>0$ had a BCR recurrence event $>24$ months after primary therapy; Figure 5B). Furthermore, we noticed that from eight patients with clinical disease 
A
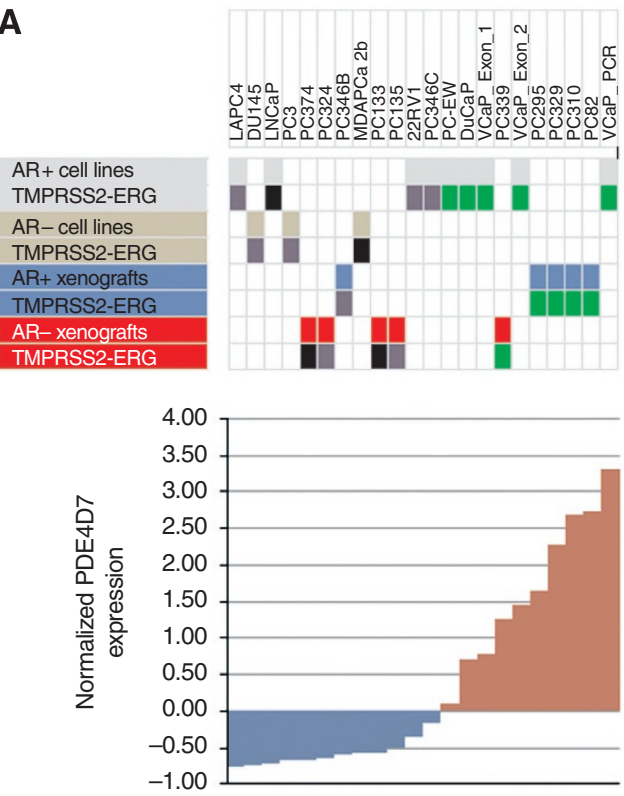

TMPRSS2-ERG negative

TMPRSS2-ERG positive

TMPRSS2-ERG negative, TMPRSS2-ETV1/ETV4 positive

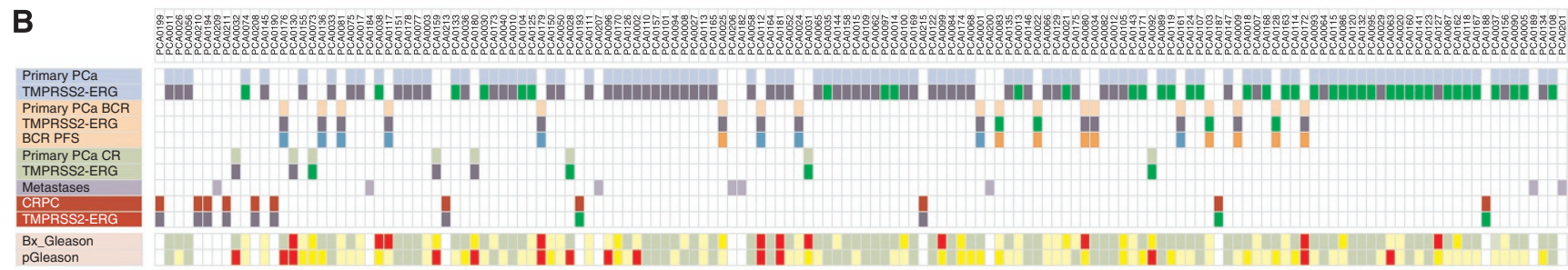

1.

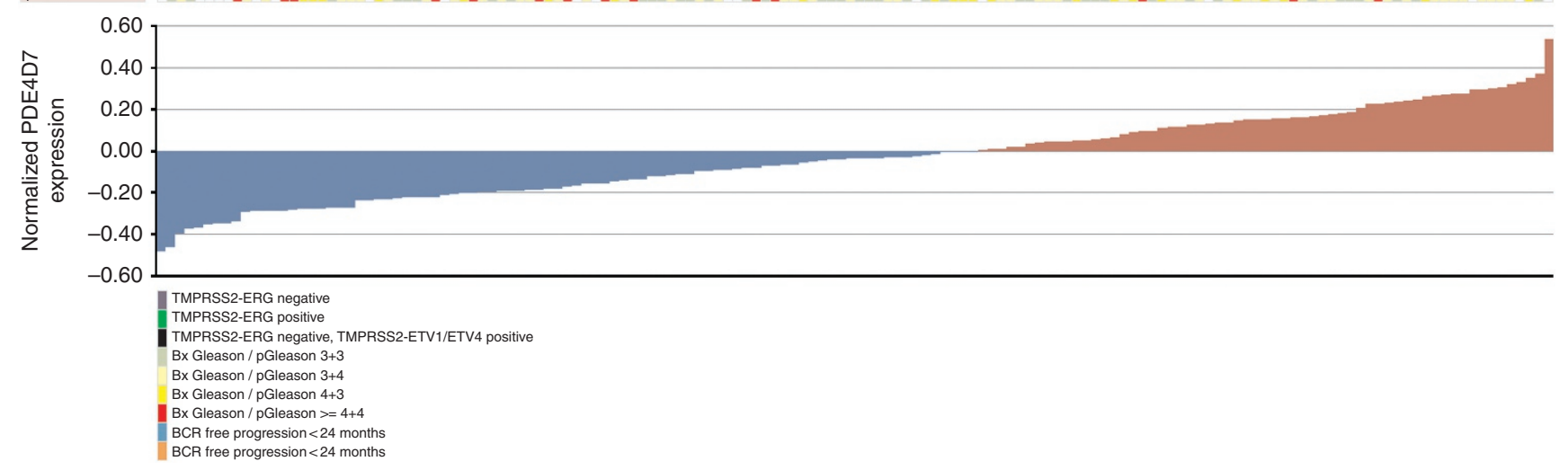

Figure 5. Correlation of PDE4D7 expression in cancerous human prostate tissues to patient outcome. A range of prostate Cancer Cell lines, xenografts, as well as patient prostate cancer tissues (data Taylor et al, 2010; Boormans et al, 2013; Böttcher et al, 2015; Supplementary Table 1) are ranked according to PDE4D7 expression in the respective cells or tissues. The normalised PDE4D7 expression value of each sample was adjusted by subtracting the mean of all expression values of the sample set. Details of cell lines, xenografts and patient samples can be found in Supplementary Table 4. (A) PDE4D7 expression in cell lines and xenograft tissues; (B) PDE4D7 expression in patient samples. For all samples its rank as well as the TMPRSS2-ERG, - ETV1 or - ETV4 fusion status is indicated. The BCR progression free survival (BCR PFS) after surgery ( $<24$ months vs $>24$ months) is indicated. Further the biopsy Gleason score (Bx_Gleason) as well as the pathology Gleason (pGleason) is given. Samples are categorised into the following groups: AR + cell lines_androgen-sensitive cell lines; AR - cell lines_androgen-insensitive cell lines; AR + xenografts—androgen-sensitive xenografts; AR - xenografts—androgen-insensitive xenografts; CRPC—castration resistant prostate cancer; metastases—-metastatic tumour; primary PCa—primary prostate cancer, no progression during follow-up; primary PCa BCR—primary prostate cancer, progression to BCR during follow-up; primary PCa CR-primary prostate cancer, progression to CR during follow-up.

recurrence during follow-up seven patients showed normalised PDE4D7 expression values $<0$ (Figure 5B) while only in one patient tissue we could measure PDE4D7 expression values $>0$ (Figure 5B).

To further confirm this, we investigated the PDE4D7 expression in samples of patients that all underwent BCR during follow-up in one data set (Taylor et al, 2010). To segregate the patients into two survival groups, we applied a PDE4D7 expression value which was derived from a ROC analysis between patients who had BCR $<24$ months $v s$ patients with $\mathrm{BCR}>24$ months. We determined the unique point of PDE4D7 expression in the ROC curve where the sum of the sensitivity and the specificity becomes a maximum 
(i.e., $<0.51)$ and used this factor for the Kaplan-Meier analysis. By this we could separate two patient cohorts $(\mathrm{HR}=0.29 ; P=6.0$ $\mathrm{E}-04$ ) with a median time to BCR after primary treatment of $<10$ months $v s$ a median time to BCR of $>30$ months (Figure 6A). When applying the same cut-off of $<0.51$ in an analysis of an independent data set (Boormans et al, 2013), we could verify this correlation to time to $\mathrm{BCR}$ after surgery $(\mathrm{HR}=0.36 ; P=1.6 \mathrm{E}-03)$ in this patient cohort with either a median time to BCR of $<10$ months, or a median time to recurrence $>50$ months (Figure 6B). The correlation of low PDE4D7 expression to time to BCR after primary treatment was further re-enforced in the second data set (Boormans et al, 2013), where time to $\mathrm{CR}$ demonstrated a fivefold increased risk of reaching the endpoint of metastatic disease within a median of 18 months after surgery when applying a cut-off $<0.26$ for PDE4D7 expression compared with a median time to $\mathrm{CR}$ of 95 months if PDE4D7 expression was $>0.26(\mathrm{HR}=0.2 ; P=2.0 \mathrm{E}-03)$ (Figure 6C). This data strongly supports our hypothesis that low expression of PDE4D7 correlates with increased short-term biochemical reoccurrence, as well as manifestation of metastatic disease.

Most samples collected from CRPC patients demonstrated low PDE4D7 expression levels while again those samples that were positive for the TMPRSS2-ERG fusion gene were measured with increased PDE4D7 transcription (Figure 5B). Whether CRPC patients with positive gene fusion and PDE4D7 expression $>0$ will survive longer compared with patients with negative TMRPSS2-ERG fusion and $\mathrm{PDE}$ expression $<0$ is a very interesting subject for further research.

\section{DISCUSSION}

Analysis of data from large scale genome sequencing projects like TCGA has uncovered a potential role of the PDE4D gene in various types of cancer (Zack et al, 2013). Indeed, loss of PDE4D was noted as one of the 10 most relevant gene deletion events in 1 study cohort (Baca et al, 2013). Although PDE4D copy number and, to a lesser degree, mutational status correlates with cancer incidence the role of PDE4D isoform expression has not been studied in a clinical context.

Recent studies have implicated individual PDE4D transcripts in the development of prostate cancer (Rahrmann et al, 2009;
Henderson et al, 2014). Specifically, we reported for the first time the downregulation of PDE4D7 in hormone-refractory prostate disease represented by a wide range of both cellular and xenograft models (Henderson et al, 2014). Here, we set out to discern whether the differential regulation of PDE4D7 could be verified in human tissue samples collected from primary, as well as metastatic and castration resistant tumours. Encouragingly, across multiple data sets we were able to detect a clear and significant downregulation of PDE4D7 transcript abundance correlating with increasing prostate disease aggressiveness (as assessed by increasing pGleason score and disease stage).

We previously demonstrated that selective knockdown of PDE4D7 expression in androgen-sensitive cell line models led to a more aggressive phenotype, while its overexpression in CRPC cells had the opposite effect (Henderson et al, 2014). The precise details of the cAMP signalling pathways regulated by PDE4D7 during the development of aggressive prostate cancer remain to be uncovered and are subject to future research. However, we would like to propose that PDE4D7 has a contributing role in initial prostate cancer cell states rather than having a 'passenger effect' occurring as a consequence of the molecular changes induced by other factors. To understand the baseline for PDE4D7 expression, and thereby contextualise the differential regulation of this particular PDE isoform during prostate cancer development and progression, we examined its expression status in normal prostate tissue compared with primary and advanced prostate cancers. Notably, the expression of the PDE4D7 transcript was significantly lower in normal, as well as tissue of benign origin compared with low-grade prostate tumours. This leads us to propose a model, where PDE4D7 expression becomes upregulated in primary disease. This, perhaps, reflects an attempt by cells to counteract the proliferative phenotype, before the failure/overcoming of this response leads to PDE4D7 downregulation, which characterises the more aggressive prostate tumours. Thus PDE4D7 appears to be functionally involved in the primary development of prostatic tumours. However, our data suggests that future cellular and molecular studies could usefully be directed to ascertain whether the initial upregulation of PDE4D7 is intimately involved in the initial stage of prostate tumorigenesis.
A

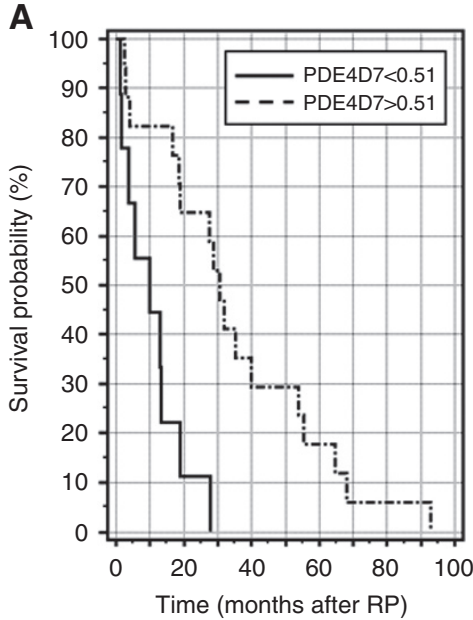

B

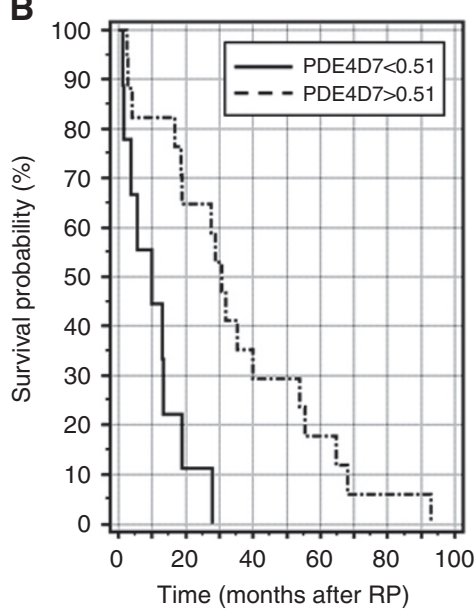

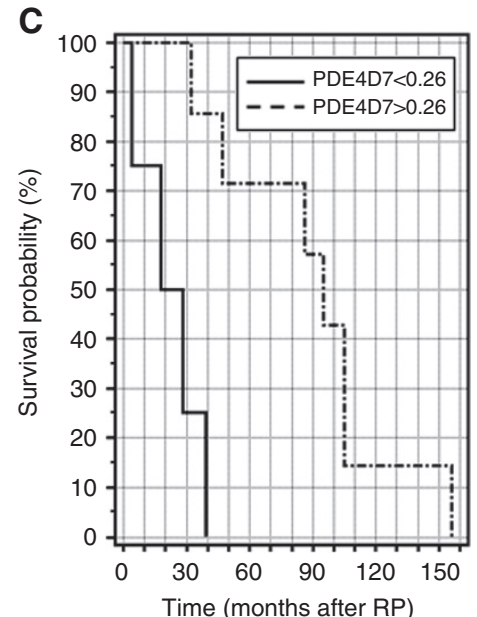

Figure 6. Kaplan-Meier survival curves in men with biochemical recurrence after primary treatment. (A and B) Using a cut-off of $<0.51$ (selected PDE4D7 expression level where the sum of the sensitivity and specificity in the ROC analysis reaches a maximum) for normalised PDE4D7 expression two patient cohorts can be separated with a median time to biochemical recurrence (BCR) after primary treatment of 9.9 and 9 months vs a median time to $\mathrm{BCR}$ of 30.6 and 50 months, respectively $(\mathrm{HR}=0.29 ; P=6.0 \mathrm{E}-04 ; \mathrm{HR}=0.36 ; P=1.6 \mathrm{E}-03$, respectively) (data: Taylor et al, 2010; Boormans et al, 2013; Böttcher et al, 2015; Supplementary Table 1). (C) Correlation of low expression of normalised PDE4D7 expression to time to BCR after primary treatment to time to clinical recurrence (data: Boormans et al, 2013). A cut-off as determined by ROC analysis of $<0.26$ for normalised PDE4D7 expression confirmed a fivefold increased risk $(H R=0.2 ; P=2.0 E-03)$ of reaching the endpoint of metastatic disease within a median of 18 months after surgery vs 95 months for PDE4D7 expression $>0.26$. 
Interestingly, we uncover here a novel link between $\mathrm{AR}$ signalling and PDE4D7 expression by correlating the incidence of TMPRSS2-ERG gene fusion and PDE4D7 transcript levels. The TMPRSS2-ERG gene fusion between the prostate specific serine protease TMPRSS2 and the ETS transcription factor family member ERG was first detected in 2005 by a statistical outlier approach (Tomlins et al, 2005). Subsequently, this gene fusion has been shown to be present in $\sim 50 \%$ of prostate cancer patients and is, consequently, one of the most prominent genomic fusion events reported in prostate cancer (Kumar-Sinha et al, 2008). This translocation results in androgen-regulated ERG expression such that the androgen-responsive promoter of TMPRSS2 now drives TMPRSS2-ERG expression, resulting in an upregulation in both the expression and activity of the transcription factor, ERG (Tomlins et al, 2005). However, despite numerous studies the clinical implications and functional consequences of the genomic fusion remain to be fully understood (Petrovics et al, 2005; Mosquera et al, 2007; Saramäki et al, 2008; Hermans et al, 2009). Here, we uncover a remarkably significant difference in PDE4D7 expression between TMPRSS2-ERG-negative and TMPRSS2-ERG-positive tumour samples. Indeed, when stratified by TMPRSS2-ERG incidence it is clear that PDE4D7 is most significantly upregulated in lowgrade TMPRSS2-ERG-positive tumours. This raises the possibility that PDE4D7 expression may be directly or indirectly regulated by the aberrant transcriptional activity of the TMPRSS2-ERG fusion protein. Inspection of the PDE4D gene reveals several putative binding sites for ERG, one within the promoter region of PDE4D7 (Materials and Methods). It would therefore seem logical that if PDE4D7 is regulated by ERG transcription, an increase in the expression of the androgen-regulated TMPRSS2-ERG factor would lead to a concurrent androgen-driven increase in PDE4D7 expression.

To date, most newly detected prostate cancer cases are clinically classified low-risk diseases (Bangma and Roobol, 2012). It is crucial to understand the natural history of these tumours as it is under considerable debate whether and to what extent low-risk Gleason 6 tumours are able to progress to higher grade tumours leading to metastatic spread or even cancer-specific death (Whittemore et al, 1991; Sowalsky et al, 2013). Interestingly, our data may indicate that reduced expression of PDE4D7 in low to intermediate Gleason tumours is correlated to progression after primary treatment. Although initially positively correlated with tumour development, the expression of PDE4D7 actually appears to be protective against further disease progression, which is in line with the data previously obtained regarding the cellular functioning of PDE4D7 (Henderson et al, 2014).

As new strategies for targeted pharmacological manipulation of specific PDE4D transcripts become available then PDE4D7 likely provides a promising future target in the treatment of primary and/ or advanced prostate cancer. Our data indicate that during tumour progression the risk of fast recurrence to clinical endpoints like biochemical or clinical disease is correlated to the level of PDE4D7 expression in the primary tumour. Consequently, patients with a low expression level of PDE4D7 in their primary cancers after surgical resection may very well be candidates for immediate adjuvant treatment like radiotherapy and/or androgen ablation. Furthermore, the manipulation of PDE4D7 suggests a strategy to selectively treat TMPRSS2-ERG fusion-positive prostate cancers. However, the success of such strategy may depend on the stratification into molecular sub-types according to the status of the TMPRSS2-ERG gene translocation.

The data presented here demonstrates the relevance of PDE4D7 as a potential biomarker for more accurate prostate cancer diagnostics. In particular, we have demonstrated the potential role of this specific splice variant of the PDE4D gene for prognosis of aggressive prostate cancer in the molecular sub-type of TMPRSS2-ERG-positive prostate tumours as well as its role as a putative target gene for therapy of primary $v s$ late-stage, hormone-refractory disease.

\section{ACKNOWLEDGEMENTS}

This study was supported by the framework of CTMM (The Netherlands), the Center for Translational Molecular Medicine, PCMM project (grant 03O-203). We would like to acknowledge support from the Biotechnology and Biological Sciences Research Council (UK) for award of a CASE studentship to DJPH. BBSRC funding to GSB (BB/G01647X/1). We also like to thank Drs Chris Bangma and Mark Wildhagen for their support regarding patient sample collection and annotation.

\section{CONFLICT OF INTEREST}

The authors declare no conflict of interest.

\section{REFERENCES}

Andriole GL, Crawford ED, Grubb RL, Buys SS, Chia D, Church TR Fouad MN, Gelmann EP, Kvale PA, Reding DJ, Weissfeld JL, Yokochi LA, O’Brien B, Clapp JD, Rathmell JM, Riley TL, Hayes RB, Kramer BS, Izmirlian G, Miller AB, Pinsky PF, Prorok PC, Gohagan JK, Berg CD (2009) Mortality results from a randomized prostate-cancer screening trial. N Engl J Med 360(13): 1310-1319.

Apuhan T, Gepdiremen S, Arslan AO, Aktas G (2013) Evaluation of patients with nasal polyps about the possible association of desmosomal junctions, RORA and PDE4D gene. Eur Rev Med Pharmacol Sci 17(19): 2680-2683.

Baca SC, Prandi D, Lawrence MS, Mosquera JM, Romanel A, Drier Y, Park K, Kitabayashi N, MacDonald TY, Ghandi M, Van Allen E, Kryukov GV, Sboner A, Theurillat J-P, Soong TD, Nickerson E, Auclair D, Tewari A, Beltran H, Onofrio RC, Boysen G, Guiducci C, Barbieri CE, Cibulskis K, Sivachenko A, Carter SL, Saksena G, Voet D, Ramos AH, Winckler W, Cipicchio M, Ardlie K, Kantoff PW, Berger MF, Gabriel SB, Golub TR, Meyerson M, Lander ES, Elemento O, Getz G, Demichelis F, Rubin MA, Garraway LA (2013) Punctuated evolution of prostate cancer genomes. Cell 153(3): 666-677.

Baillie GS, MacKenzie SJ, McPhee I, Houslay MD (2000) Sub-family selective actions in the ability of Erk2 MAP kinase to phosphorylate and regulate the activity of PDE4 cyclic AMP-specific phosphodiesterases. $\mathrm{Br} J$ Pharmacol 131(4): 811-819.

Bangma CH, Roobol MJ (2012) Defining and predicting indolent and low risk prostate cancer. Crit Rev Oncol 83(2): 235-241.

Böttcher R, Hoogland AM, Dits N, Verhoef EI, Kweldam C, Waranecki P, Bangma CH, van Leenders GJ, Jenster G (2015) Novel long non-coding RNAs are specific diagnostic and prognostic markers for prostate cancer. Oncotarget 6(6): 4036-4050.

Boormans JL, Korsten H, Ziel-van der Made AJC, van Leenders GJLH, de Vos CV, Jenster G, Trapman J (2013) Identification of TDRD1 as a direct target gene of ERG in primary prostate cancer. Int J Cancer 133(2): 335-345.

Brase J, Johannes M, Mannsperger H, Falth M, Metzger J, Kacprzyk L, Andrasiuk T, Gade S, Meister M, Sirma H, Sauter G, Simon R, Schlomm T, BeiSZbarth T, Korf U, Kuner R, Sultmann H (2011) TMPRSS2-ERG specific transcriptional modulation is associated with prostate cancer biomarkers and TGF-beta signaling. BMC Cancer 11(1): 507.

Byrne AM, Elliott C, Hoffmann R, Baillie GS (2015) The activity of cAMPphosphodiesterase 4D7 (PDE4D7) is regulated by protein kinase A-dependent phosphorylation within its unique N-terminus. FEBS Lett 589(6): 750-755.

Conti M, Beavo J (2007) Biochemistry and physiology of cyclic nucleotide phosphodiesterases: essential components in cyclic nucleotide signaling. Annu Rev Biochem 76(1): 481-511.

Erho N, Crisan A, Vergara IA, Mitra AP, Ghadessi M, Buerki C, Bergstralh EJ, Kollmeyer T, Fink S, Haddad Z, Zimmermann B, Sierocinski T, Ballman KV, Triche TJ, Black PC, Karnes RJ, Klee G, Davicioni E, Jenkins RB (2013) Discovery and validation of a prostate cancer genomic classifier that predicts early metastasis following radical prostatectomy. PLoS One 8(6): e66855.

Ferlay J, Soerjomataram I, Dikshit R, Eser S, Mathers C, Rebelo M, Parkin DM, Forman D, Bray F (2015) Cancer incidence and mortality worldwide: sources, methods and major patterns in GLOBOCAN 2012. Int J Cancer 136(5): E359-E386. 
Francis SH, Blount MA, Corbin JD (2011) Mammalian cyclic nucleotide phosphodiesterases. Physiol Rev 91(5): 651-690.

Gretarsdottir S, Thorleifsson G, Reynisdottir ST, Manolescu A, Jonsdottir S, Jonsdottir T, Gudmundsdottir T, Bjarnadottir SM, Einarsson OB, Gudjonsdottir HM, Hawkins M, Gudmundsson G, Gudmundsdottir H, Andrason H, Gudmundsdottir AS, Sigurdardottir M, Chou TT, Nahmias J, Goss S, Sveinbjornsdottir S, Valdimarsson EM, Jakobsson F, Agnarsson U, Gudnason V, Thorgeirsson G, Fingerle J, Gurney M, Gudbjartsson D, Frigge ML, Kong A, Stefansson K, Gulcher JR (2003) The gene encoding phosphodiesterase 4D confers risk of ischemic stroke. Nat Genet 35(2): 131-138.

Henderson DJP, Byrne A, Dulla K, Jenster G, Hoffmann R, Baillie GS, Houslay MD (2014) The cAMP phosphodiesterase-4D7 (PDE4D7) is downregulated in androgen-independent prostate cancer cells and mediates proliferation by compartmentalising cAMP at the plasma membrane of VCaP prostate cancer cells. Br J Cancer 110(5): 1278-1287.

Hermans KG, Boormans JL, Gasi D, van Leenders GJHL, Jenster G, Verhagen PCMS, Trapman J (2009) Overexpression of prostate-specific TMPRSS2(exon 0)-ERG fusion transcripts corresponds with favorable prognosis of prostate cancer. Clin Cancer Res 15(20): 6398-6403.

Hoffmann R, Baillie GS, MacKenzie SJ, Yarwood SJ, Houslay MD (1999) The MAP kinase ERK2 inhibits the cyclic AMP-specific phosphodiesterase HSPDE4D3 by phosphorylating it at Ser579. $E M B O J$ 18(20): 893-903.

Hoffmann R, Wilkinson IR, McCallum JF, Engels P, Houslay MD (1998) cAMP-specific phosphodiesterase HSPDE4D3 mutants which mimic activation and changes in rolipram inhibition triggered by protein kinase A phosphorylation of Ser-54: generation of a molecular model. Biochem J 333(Pt 1): 139-149.

Houslay MD (2010) Underpinning compartmentalised cAMP signalling through targeted cAMP breakdown. Trends Biochem Sci 35(2): 91-100.

Houslay MD, Baillie GS, Maurice DH (2007) cAMP-Specific phosphodiesterase- 4 enzymes in the cardiovascular system: a molecular toolbox for generating compartmentalized cAMP signaling. Circ Res 100(7): 950-966.

Houslay MD, Schafer P, Zhang KYJ (2005) Keynote review: phosphodiesterase-4 as a therapeutic target. Drug Discov Today 10(22): 1503-1519.

Kaname T, Ki C-S, Niikawa N, Baillie GS, Day JP, Yamamura K-i, Ohta T, Nishimura G, Mastuura N, Kim O-H, Sohn YB, Kim HW, Cho SY, Ko A-R, Lee JY, Kim HW, Ryu SH, Rhee H, Yang K-S, Joo K, Lee J, Kim CH, Cho K-H, Kim D, Yanagi K, Naritomi K, Yoshiura K-i, Kondoh T, Nii E, Tonoki H, Houslay MD, Jin D-K (2014) Heterozygous mutations in cyclic AMP phosphodiesterase-4D (PDE4D) and protein kinase A (PKA) provide new insights into the molecular pathology of acrodysostosis. Cell Signal 26(11): 2446-2459.

Kumar-Sinha C, Tomlins SA, Chinnaiyan AM (2008) Recurrent gene fusions in prostate cancer. Nat Rev Cancer 8(7): 497-511.

Lee H, Graham John M, Rimoin David L, Lachman Ralph S, Krejci P, Tompson Stuart W, Nelson Stanley F, Krakow D, Cohn Daniel H (2012) Exome sequencing identifies PDE4D mutations in acrodysostosis. Am J Hum Genet 90(4): 746-751.

Li B, Ruotti V, Stewart RM, Thomson JA, Dewey CN (2010) RNA-Seq gene expression estimation with read mapping uncertainty. Bioinformatics 26(4): 493-500.

Lugnier C (2006) Cyclic nucleotide phosphodiesterase (PDE) superfamily: a new target for the development of specific therapeutic agents. Pharmacol Therap 109(3): 366-398.

MacKenzie KF, Wallace DA, Hill EV, Anthony DF, Henderson DJP, Houslay DM, Arthur JSC, Baillie GS, Houslay MD (2011) Phosphorylation of cAMP-specific PDE4A5 (phosphodiesterase-4A5) by MK2 (MAPKAPK2) attenuates its activation through protein kinase A phosphorylation. Biochem J 435(Article): 755-769.

Maurice DH, Ke H, Ahmad F, Wang Y, Chung J, Manganiello VC (2014) Advances in targeting cyclic nucleotide phosphodiesterases. Nat Rev Drug Discov 13(4): 290-314.

Merkle D, Hoffmann R (2011) Roles of cAMP and cAMP-dependent protein kinase in the progression of prostate cancer: Cross-talk with the androgen receptor. Cell Signal 23(3): 507-515.

Michot C, Le Goff C, Goldenberg A, Abhyankar A, Klein C, Kinning E, Guerrot A-M, Flahaut P, Duncombe A, Baujat G, Lyonnet S,
Thalassinos C, Nitschke P, Casanova J-L, Le Merrer M, Munnich A, Cormier-Daire V (2012) Exome sequencing identifies PDE4D mutations as another cause of acrodysostosis. Am J Hum Genet 90(4): 740-745.

Mosquera JM, Perner S, Demichelis F, Kim R, Hofer MD, Mertz KD, Paris PL, Simko J, Collins C, Bismar TA, Chinnaiyan AM, Rubin MA (2007) Morphological features of TMPRSS2-ERG gene fusion prostate cancer. J Pathol 212(1): 91-101.

Petrovics G, Liu A, Shaheduzzaman S, Furusato B, Sun C, Chen Y, Nau M, Ravindranath L, Chen Y, Dobi A, Srikantan V, Sesterhenn IA, McLeod DG, Vahey M, Moul JW, Srivastava S (2005) Frequent overexpression of ETS-related gene-1 (ERG1) in prostate cancer transcriptome. Oncogene 24(23): 3847-3852.

Purdom E, Simpson KM, Robinson MD, Conboy JG, Lapuk AV, Speed TP (2008) FIRMA: a method for detection of alternative splicing from exon array data. Bioinformatics 24(15): 1707-1714.

Rahrmann EP, Collier LS, Knutson TP, Doyal ME, Kuslak SL, Green LE, Malinowski RL, Roethe L, Akagi K, Waknitz M, Huang W, Largaespada DA, Marker PC (2009) Identification of PDE4D as a proliferation promoting factor in prostate cancer using a Sleeping Beauty transposon based somatic mutagenesis screen. Cancer Res 69(10): 4388-4397.

Saramäki OR, Harjula AE, Martikainen PM, Vessella RL, Tammela TLJ, Visakorpi T (2008) TMPRSS2:ERG fusion identifies a subgroup of prostate cancers with a favorable prognosis. Clin Cancer Res 14(11): 3395-3400.

Schröder FH, Hugosson J, Roobol MJ, Tammela TLJ, Ciatto S, Nelen V, Kwiatkowski M, Lujan M, Lilja H, Zappa M, Denis LJ, Recker F, Berenguer A, Määttänen L, Bangma CH, Aus G, Villers A, Rebillard X, van der Kwast T, Blijenberg BG, Moss SM, de Koning HJ, Auvinen A (2009) Screening and prostate-cancer mortality in a randomized european study. N Engl J Med 360(13): 1320-1328.

Sheppard CL, Lee LCY, Hill EV, Henderson DJP, Anthony DF, Houslay DM, Yalla KC, Cairns LS, Dunlop AJ, Baillie GS, Huston E, Houslay MD (2014) Mitotic activation of the DISC1-inducible cyclic AMP phosphodiesterase4D9 (PDE4D9), through multi-site phosphorylation, influences cell cycle progression. Cell Signal 26(9): 1958-1974.

Sowalsky AG, Ye H, Bubley GJ, Balk SP (2013) Clonal progression of prostate cancers from gleason grade 3 to grade 4. Cancer Res 73(3): 1050-1055.

Taylor BS, Schultz N, Hieronymus H, Gopalan A, Xiao Y, Carver BS, Arora VK, Kaushik P, Cerami E, Reva B, Antipin Y, Mitsiades N, Landers T, Dolgalev I, Major JE, Wilson M, Socci ND, Lash AE, Heguy A, Eastham JA, Scher HI, Reuter VE, Scardino PT, Sander C, Sawyers CL Gerald WL (2010) Integrative genomic profiling of human prostate cancer. Cancer Cell 18(1): 11-22.

Tomlins SA, Rhodes DR, Perner S, Dhanasekaran SM, Mehra R, Sun X-W, Varambally S, Cao X, Tchinda J, Kuefer R, Lee C, Montie JE, Shah RB, Pienta KJ, Rubin MA, Chinnaiyan AM (2005) Recurrent fusion of TMPRSS2 and ETS transcription factor genes in prostate cancer. Science 310(5748): 644-648.

Wang D, Deng C, Bugaj-Gaweda B, Kwan M, Gunwaldsen C, Leonard C Xin X, Hu Y, Unterbeck A, De Vivo M (2003) Cloning and characterization of novel PDE4D isoforms PDE4D6 and PDE4D7. Cell Signal 15(9): 883-891.

Whittemore AS, Keller JB, Betensky R (1991) Low-grade, latent prostate cancer volume: predictor of clinical cancer incidence? J Natl Cancer Inst 83(17): 1231-1235.

Yoon H-K, Hu H-J, Rhee C-K, Shin S-H, Oh Y-M, Lee S-D, Jung S-H, Yim S-H, Kim T-M, Chung Y-J (2014) Polymorphisms in PDE4D are associated with a risk of COPD in non-emphysematous koreans. COPD 11(6): 652-658.

Zack TI, Schumacher SE, Carter SL, Cherniack AD, Saksena G, Tabak B, Lawrence MS, Zhang C-Z, Wala J, Mermel CH, Sougnez C, Gabriel SB, Hernandez B, Shen H, Laird PW, Getz G, Meyerson M, Beroukhim R (2013) Pan-cancer patterns of somatic copy number alteration. Nat Genet 45(10): 1134-1140.

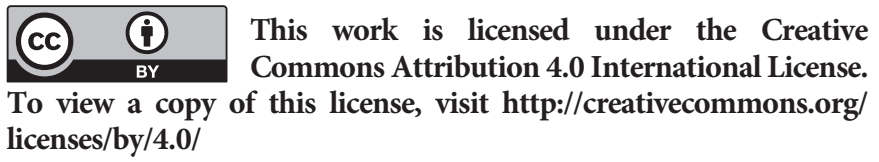

This work is licensed under the Creative Commons Attribution 4.0 International License. licenses/by/4.0/

Supplementary Information accompanies this paper on British Journal of Cancer website (http://www.nature.com/bjc) 